\title{
T. Kobayashi, L.D. Lunsford (eds): Pineal region tumors-diagnosis and treatment options. (Progress in neurological surgery, vol. 23)
}

\author{
Karger, Basel, 2009. X, 146 pp, 58 figures, 28 tables (ISBN 978-3-8055-9077-8)
}

\author{
Concezio Di Rocco
}

Published online: 1 August 2009

(C) Springer-Verlag 2009

The pineal gland has longly attracted the attention of anatomists and philosophers as its deep location in the middle of the brain and its apparent "protection" by the surrounding structures seemed to indicate a particularly important function. In a similar way, the topographic arrangement of the gland has stimulated the neurosurgeon to develop surgical approaches utilizing natural corridors between the two cerebral hemispheres or taking advantage from its proximity to the third ventricle. In the course of the years, these approaches have became more safe, precise, and effective allowing the direct excision of tumors in the regions in many cases. The advent of endoscopy has also allowed to obtain the diagnosis of nature for lesions not amenable to the surgical treatment but responsive to radiotherapy and chemotherapy. Consequently, the treatment of pineal region tumors has became nowadays less elusive.

This book, edited by T. Kobayashi and L.D. Lunsford aims at reviewing some aspects, namely related to the classification, the diagnosis and treatment strategies of the numerous and different tumors which may originate in the pineal region, taking advantage of the experience matured in Japan, where these lesions tend to be more often encountered. Actually, with the exception of a chapter co-edited by Lunsford concerning stereotactic radiosurgery of pineal parenchymal tumor, all the other chapters of the book are written by Japanese neurosurgeons. Also te

\section{Di Rocco $(\bowtie)$}

Section of Pediatric Neurosurgery, Department of Neurosurgery, Catholic University, Medical School,

00168 Rome, Italy

e-mail: cdirocco@rm.unicatt.it introductory chapter dealing with the incidence of pineal tumors is based on the data of the Japanese Brain tumors registry instituted in 1975 and to which currently cooperate 473 neurosurgical institutions. This chapter concerns primary brain tumors registered in Japan between 1984 and 2000 and confirms that germ cell tumors with a relative incidence of $14.6 \%$ account for a high percentage of neoplasms in this country where glioma represents $25-6 \%$ of all primary brain tumors.

Then, the core of the book is subdivided into two main sections: Tumors of Pineal Cell origin and Tumors of Germ Cell origin. The architecture of these two sections is quite traditional, based on an introductory chapter on the pathology and then specific contributions on the various surgical, radiotherapic, radiosurgical, and stereotactic radiosurgical treatments. An interesting final chapter dealing with the quality of life in long-term survivors after radiotherapy demonstrates the effectiveness of the treatment in terms of survival but also the known deterioration in cognitive performance in most of the survivors, with a high percentage of them unable to get or to maintain an occupation.

In conclusion, this book certainly reaches its main goal to provide the state of art in the treatment of these rare tumors in a country, like Japan, in which their incidence is relatively high.

Obviously, the data collected are useful also to neurosurgeons, oncologists and radiotherapists working elsewhere. The quality of printing is excellent and the numerous figures, several of them in color, scattered throughout the text contribute to make the book particularly readable. 